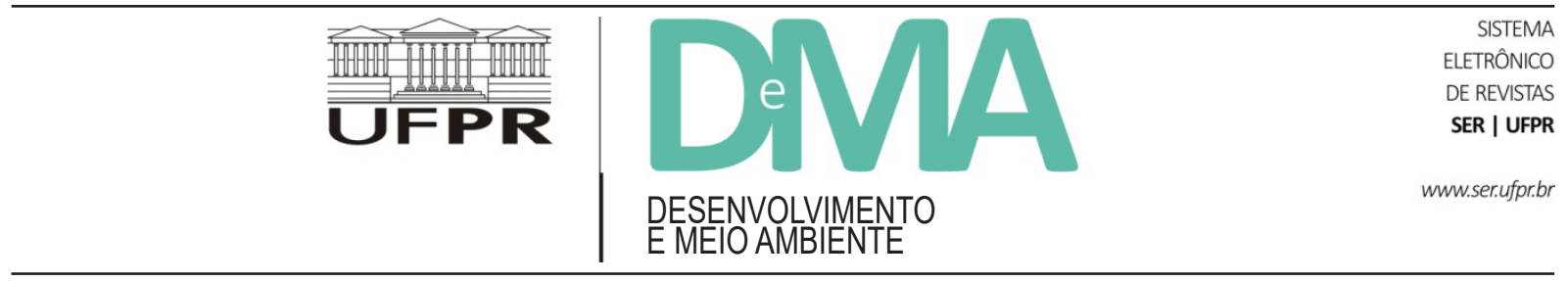

\title{
Agroecología en la periferia: un caso del territorio maya-achí, Guatemala ${ }^{1}$
}

\section{Agroecologia na periferia: um caso do território Maya-Achi, Guatemala}

\section{Agroecology on the periphery: A case from the Maya-Achí territory, Guatemala}

\author{
Nathan EINBINDER ${ }^{1 *}$, Helda MORALES ${ }^{1}$, Mateo MIER y TERÁN Gimenéz Cacho ${ }^{1}$, Miriam ALDASORO ${ }^{1}$, \\ Bruce FERGUSON ${ }^{1}$, Ronald $\mathrm{NIGH}^{2}$ \\ ${ }^{1}$ El Colegio de la Frontera Sur (ECOSUR), San Cristóbal de Las Casas, Chiapas, México. \\ ${ }^{2}$ Centro de Investigaciones y Estudios Superiores de Antropología Social, Ciudad del México, DF, México. \\ *E-mail de contacto: nathaneinbinder@gmail.com
}

Artículo recibido en 11 de junio de 2021, publicado en 30 de noviembre de 2021.

RESUMEN: En este documento examina la ampliación de las prácticas agroecológicas en el territorio maya achí de Guatemala. Comparamos este caso con otros documentados en la literatura, así como los factores clave o "impulsores" importantes para su escalamiento. Hemos constatado la complejidad de sus factores impulsores y que estos, como los métodos constructivistas de aprendizaje/enseñanza, las políticas públicas favorables y el fuerte tejido social, parecen ser débiles, ausentes o incluso negativos, lo que en parte se debe a la violencia y la represión de los años ochenta, que resultó en el asesinato del $20 \%$ de la población dejando el territorio socialmente fragmentado. En este marco, los proyectos que incorporan la agroecología se consideran una estrategia potencial para la recuperación de la comunidad, de modo que son promovidos por asociaciones locales, instituciones y ONG internacionales. Si bien en un principio se planteó que la recuperación social y cultural era la causa principal de la adopción de tales prácticas, nos encontramos con distintos factores adicionales y complejos,

\footnotetext{
${ }^{1}$ Este artículo fue publicado originalmente en inglés en: Einbinder, N.; Morales, H., Mier y Terán, M. G. C.; Aldasoro, M., Ferguson, B. G.; Nigh, R. Agroecology on the periphery: A case from the Maya-Achí territory, Guatemala. Agroecology and Sustainable Food Systems, 43(7-8), 744-763, 2019.
} 
entre estos, la expectativa de beneficios económicos y la presencia de organizaciones de ayuda y desarrollo. Analizando estos factores y las barreras contribuimos al debate en curso sobre cómo se pueden ampliar las prácticas agroecológicas, en particular en las regiones que no tienen las condiciones ideales.

Palavras-chave: agroecología; Maya-Achí; Guatemala; organizaciones no governamentales; desarollo.

RESUMO: Neste documento, examinamos os processos de escalada das práticas agroecológicas no território maia-aquí da Guatemala. Comparamos o caso Achí com outros exemplos documentados na literatura e com os fatores-chave, ou "impulsores", relatados como importantes, se não essenciais, para que a escalada ocorra. Descobrimos que o caso Achí é complexo no que diz respeito a esses motoristas. Fatores como os métodos construtivistas de aprendizagem/ensino, políticas públicas favoráveis e tecido social forte parecem ser fracos, ausentes ou mesmo negativos. Isto se deve em parte à violência e repressão dos anos 80 , que resultou no assassinato de $20 \%$ da população pelos militares e paramilitares, deixando o território socialmente fragmentado. Projetos incorporando a agroecologia (revalorização de práticas ancestrais, economia de sementes, eliminação de insumos externos, fortalecimento da saúde do solo, aumento/guarda da agrobiodiversidade) são vistos como uma estratégia potencial para ajudar na recuperação da comunidade, e são promovidos por associações locais, assim como por instituições internacionais e ONGs. Enquanto as recuperações social e cultural foram inicialmente hipotéticas como causas primárias para a adoção de práticas, encontramos uma série de fatores adicionais e complexos, tais como a expectativa de benefícios econômicos e a presença de organizações de ajuda e desenvolvimento. Analisando esses fatores e barreiras, contribuímos para o debate em curso sobre como as práticas agroecológicas podem ser escalonadas, particularmente em regiões que exibem condições menos que ideais.

Palavras-chave: agroecologia; Maya-Achí; Guatemala; organizações não governamentais; desenvolvimento.

ABSTRACT: In this paper we examine processes of scaling agroecological practices in the Maya-Achí territory of Guatemala. We compare the Achí case to other examples documented in the literature and the key factors, or "drivers," reported as important if not essential for scaling to occur. We find that the Achí scase is complex with regard to these drivers. Factors such as constructivist learning/teaching methods, favorable public policies, and strong social fabric appear to be weak, absent, or even negative. This is due in part to the violence and repression of the $1980 \mathrm{~s}$, which resulted in the assassination of 20 percent of the population by the military and paramilitaries, leaving the territory socially fragmented. Projects incorporating agroecology (revalorization of ancestral practices, seed saving, elimination of external inputs, strengthening soil health, increasing/guarding agrobiodiversity) are viewed as a potential strategy to aid in community recovery, and are promoted by local associations as well as by international institutions and NGOs. While social and cultural recuperation were initially hypothesized as primary causes for the adoption of practices, we encounter a range of additional and complex factors, such as the expectation of economic benefits and the presence of aid and development organizations. By analyzing these drivers and barriers we contribute to the ongoing debate over how agroecological practices may be scaled-out, particularly in regions exhibiting less than ideal conditions.

Keywords: Agroecology; Maya-Achí; Guatemala; non-governmental organizations; development. 


\section{Introducción}

Guatemala, junto con gran parte de la región mesoamericana, tiene fuertes vínculos presentes e históricos con el uso de las prácticas agroecológicas (Wilken, 1987; Morales \& Perfecto, 2000). Y una contribución clave a nuestro conocimiento de esa agricultura sostenible y diversificada es la milpa tradicional (Isakson, 2009). Desarrollada a lo largo de siglos, este agroecosistema de policultivo, compuesto por variedades locales de maíz, frijoles, calabaza y verduras nativas, sigue siendo la base de la dieta, la cultura y la economía rural mesoamericanas (Steinberg, 1999; IDEAR, 2010).

Asimismo, la región se destaca por sus profundas conexiones con el "movimiento" agroecológico; es decir, con las acciones sociales y políticas que impulsan la difusión de prácticas y principios agrícolas sostenibles/ancestrales, a menudo conectados con luchas territoriales más amplias (Rosset \& Martínez-Torres, 2012; McCune \& Sánchez, 2018). Como ilustra HoltGiménez (2006), a lo largo de los años setenta y ochenta miles de campesinos reclamaron la autonomía a nivel familiar y comunitario mediante el intercambio horizontal de conocimientos y prácticas agroecológicas, un proceso metodológico de aprendizaje y acción ahora denominado Campesino a Campesino $(\mathrm{CaC})$.

Los resultados fueron impresionantes, no solo en lo que respecta al número de participantes, sino también en la transformación y el mejoramiento a largo plazo de sus campos, mediante el uso de abonos verdes y otras técnicas fácilmente apropiables (Wettasinha et al., 2014). Sin embargo, como parte de una tendencia más amplia que suponía la eliminación de los movimientos sociales por parte de los gobiernos de derecha, la incipiente transformación agroecológica se interrumpió. En Guatemala, actualmente en el ápice de su conflicto armado de 36 años de duración, la búsqueda de la independencia y la autosuficiencia de los campesinos fueron consideradas por los militares como equivalentes a la "subversión"; entonces, además de una política para su erradicación, se puso en marcha una campaña para desmantelar el liderazgo y su base por todos los medios necesarios (Jonas, 1991; CEH, 1999).

De hecho, una gran cantidad de investigaciones siguen este caso ejemplar, desde su influencia en los territorios vecinos (Machín Sosa et al., 2013; Rosset, 2014) hasta la documentación de otros ejemplos en el mundo en los que se está utilizando y construyendo el modelo $\mathrm{CaC}$ (Khadse et al., 2017). Recientemente, varios estudios tratan de caracterizar los movimientos agroecológicos eficaces en todo el mundo, a fin de abordar las preocupaciones sobre el porqué las prácticas y los principios sostenibles no se han difundido mayormente, pese al amplio apoyo internacional y las pruebas científicas (Parmentier, 2014; Brescia, 2017; Khadse et al., 2017). Mier y Terán et al. (2018) contribuyen al debate analizando estudios de casos emblemáticos en los que las prácticas agroecológicas se han escalado, incluido el $\mathrm{CaC}, \mathrm{y}$ han generado una lista de factores compartidos o "impulsores": 1) reconocimiento de una crisis que motiva la búsqueda de alternativas, 2) organización social, 3) procesos de aprendizaje constructivista, 4) prácticas agroecológicas efectivas, 5) movilización de discursos, 6) aliados externos, 7) mercados favorables y 8) políticas favorables. 
En este artículo se examina el escalamiento agroecológico en el territorio maya achí guatemalteco. Y si bien hay varias características de este caso que lo unen con el $\mathrm{CaC}$, en lo concerniente a la historia cultural y colonial compartida, se distingue por su complejidad. Lo que más nos preocupa son las consecuencias persistentes del conflicto armado. Como uno de los cuatro lugares en los que se confirmó el genocidio patrocinado por el Estado (CEH, 1999), el territorio achí todavía se recupera de la pérdida de más del $20 \%$ de su población, de la desaparición de líderes y la completa ruptura de las relaciones sociales (Stewart, 2006; Einbinder, 2017). Influyen, asimismo, factores como las sequías recurrentes debidas al cambio climático, y patrones y valores de consumo que cambian rápidamente.

Al comparar nuestro caso de análisis con las teorías y debates más amplios en torno al escalamiento agroecológico, hemos hallado una serie de inconsistencias. Específicamente nos referimos a la ausencia de un fuerte tejido social, apoyo político y métodos constructivistas de aprendizaje/enseñanza. Como se ha señalado, todos estos factores son impulsores importantes, si no es que esenciales, en la adopción de las prácticas agroecológicas. Detectamos también un conjunto mucho más complejo de impactos resultantes de la presencia de organizaciones y alianzas externas que los que se encuentran en la literatura.

A partir de estas observaciones, junto con lo confirmado en el territorio como actividad significativa en el escalamiento agroecológico - con cientos de familias involucradas y una gran cantidad de organizaciones e intereses participantes- identificamos el caso achí como único en comparación con otros. Nuestro objetivo en este texto es demostrar cómo puede ocurrir el escalamiento agroecológico en ausencia de impulsores clave como se indica en la literatura, así como ampliar la discusión sobre los impactos positivos y negativos de los aliados externos. Para ello, presentamos el contexto social e histórico, seguido de la identificación de barreras, impulsores y actores exclusivos del caso. Nos proponemos abrir nuevos puntos de discusión y debate para contribuir críticamente a la teoría en desarrollo.

\section{Métodos de investigación}

El trabajo de campo se llevó a cabo entre febrero de 2017 y julio de 2018; no obstante, hubo un proceso más amplio de acompañamiento e investigación. Durante este periodo, el autor principal pasó ocho meses reuniendo datos cualitativos mediante entrevistas semiestructuradas, acompañamiento a 14 productores agroecológicos -en su mayoría miembros de la asociación de agricultores Qachuu Aloom-, y observación participante (Dunn, 2005; DeWalt \& DeWalt, 2011). Los entrevistados fueron seleccionados como parte de un proceso participativo/colaborativo con los promotores locales (Guzmán \& Alonso, 2007), quienes también ayudaron en el desarrollo de las preguntas, la traducción y la logística. Los entrevistados incluyen directores y empleados de organizaciones que trabajan en la región, muchos de ellos productores agroecológicos. Finalmente, realizamos una amplia revisión de la literatura, así como el análisis de los informes de los proyectos, desarrollando una "triangulación" de métodos para asegurar el rigor (Baxter \& Eyles, 1997). 


\subsection{Consideraciones teóricas}

Esta investigación se fundamenta en el marco crítico de la agroecología, en el que los aspectos técnicos interactúan con la investigación del papel de la sociedad y su transición hacia la sostenibilidad utilizando prácticas y principios agroecológicos (Pretty, 2002; Gliessman, 2015; Perfecto \& Vandemeer, 2015). En tanto fusión de los conocimientos y técnicas indígenas/tradicionales (Deneven, 1995; Morales \& Perfecto, 2000) con la ciencia contemporánea (Altieri, 1987; Hainzelen, 2014), las prácticas agroecológicas están arraigadas en la diversidad y la economía. Aludimos en específico al uso económico e innovador del espacio y los recursos, como el suelo, el agua, los nutrientes, los árboles y otras plantas, el material genético y la mano de obra (Nichols et al., 2016). Según estas directrices, las prácticas agroecológicas tratan de eliminar los insumos externos usando materia orgánica y desechos animales para mejorar el suelo; de utilizar la biodiversidad para la gestión de plagas; de reducir la dependencia y aumentar la adaptabilidad conservando las semillas del patrimonio, y de recuperar la nutrición de las casas/comunidades y la seguridad alimentaria diversificando los modos de producción (Sarandón \& Flores, 2014).

Como enfoque teórico, la agroecología desafía la lógica y los mecanismos que subyacen a la revolución verde (Kremen et al., 2012), y a las narraciones sobre la necesidad de aumentar la producción (Perfecto et al., 2009); enfrenta la insostenibilidad del sistema alimentario mundial (Weis, 2007), al tiempo que demuestra el potencial de resistencia y adaptabilidad de los sistemas agroecológicos, en particular entre los agricultores en pequeña escala $\mathrm{y} / \mathrm{o}$ familiares más aptos para utilizarlos y desarrollarlos (Holt-Gimenez, 2002). Como lo resume Gliessman \& Rosemeyer (2010), la agroecología responde a las necesidades locales, a menudo comenzando por el productor que rediseña su parcela en función de las microcondiciones, la disponibilidad de recursos y la pertinencia cultural; pero también aspira a transformar las relaciones entre los agentes del sistema alimentario desde el nivel local al mundial. Así pues, la agroecología va más allá de los pilares de la producción orgánica y diversificada y entra en el ámbito de los procesos sociales, los mercados, la educación, la restauración ecológica y el desarrollo (Wezel et al., 2009; Altieri \& Toledo, 2011).

En este marco, nuestra investigación parte de preocupaciones teóricas relativas a la escala agroecológica, también denominada "masificación", "transformación" o "reconfiguración" agroecológica (González, 2012). Para ello, definimos el escalamiento como la difusión horizontal y la adopción de prácticas y principios agroecológicos en todo el espacio geográfico. De la literatura al respecto, llama la atención en particular el énfasis en la organización social, es decir, en el denominado "medio de cultivo en el cual crece la agroecología" (Mier y Terán et al. 2018, p. 19). Adicionalmente tomamos en cuenta los discursos del alto nivel de incidencia e importancia de los métodos constructivistas de aprendizaje y enseñanza, el apoyo de aliados externos y las políticas públicas favorables. Nuestro interés por estos impulsores específicos se relaciona precisamente con el hecho de que, en nuestro caso, o bien están parcial y/o totalmente ausentes, o bien, con respecto a los aliados externos, generan muchas más preguntas de 
las que se describen en la literatura. En los siguientes apartados ilustramos el movimiento agroecológico que se está produciendo en el territorio achí para contribuir al debate teórico.

\subsection{Contexto geográfico}

El territorio maya achí se encuentra en el departamento rural de las tierras altas de Baja Verapaz, aproximadamente a $145 \mathrm{~km}$ al norte de la ciudad de Guatemala. Con aproximadamente 100 mil habitantes indígenas que hablan el idioma achí (SEGEPLAN, 2010), el territorio abarca tres municipios principales, el más grande es Rabinal, cuya capital sirve como centro político y económico. La industria local tiene como actividades preponderantes la artesanía y la agricultura, esta última centrada en la subsistencia y la infrasubsistencia, principalmente en la milpa, pero también en la producción de hortalizas en pequeña escala, cacahuate y café. Conocidos como la cuna folclórica de la nación, los achí se distinguen por la preservación de las ceremonias y rituales prehispánicos (Tedlock, 2003), por el uso continuo de la vestimenta y el lenguaje tradicionales, así como por el apego a la tierra y una gastronomía distinta basada en bebidas calientes de maíz (atol), calabaza local (ayote) y verduras semisalvajes (Acevedo, 2004; Luna-Gonzáles \& Sørenson, 2018).

Si bien extraordinario por la preservación de sus prácticas culturales, una investigación más profunda revela un pueblo marcado por la dominación, la violencia y la exclusión; concretamente nos referimos al último ciclo de "conquista" (Lovell, 1988), cuando fue objeto de una campaña de eliminación patrocinada por el gobierno (CIIDH, 1999). Se podría escribir mucho sobre el conflicto armado guatemalteco y su impacto en la sociedad contemporánea, pero dejamos los detalles a otras fuentes (Carmack, 1988; Jonas, 1991; Ball et al., 1999; CEH, 1999; CIIDH, 1999; Benson et al., 2008). No obstante, para los que no están familiarizados, cabe señalar que más de 200 mil civiles, en su mayoría indígenas, fueron asesinados en un periodo de 36 años, entre 1960 y 1996; un gran porcentaje a principios de los años ochenta bajo las dictaduras militares sancionadas por Estados Unidos. Sus causas fundamentales estaban relacionadas con las pautas arraigadas de exclusión social del país, el racismo étnico y una política de desarrollo destinada a beneficiar a una selecta élite minoritaria. La promesa de reforma democrática del presidente Jacobo Árbenz Guzmán, y su derrocamiento en 1954 por parte de la Agencia Central de Inteligencia (CIA, por sus siglas en inglés), se citan ampliamente como instigadores adicionales. Hasta el día de hoy Árbenz sigue siendo un símbolo de esperanza perdida.

En el territorio achí el ejército tomó medidas preventivas contra una población juzgada vulnerable al adoctrinamiento de los llamados subversivos, usando para ello todo el potencial de las Patrullas de Autodefensa Civil (PAC) (CEH, 1999). Las PAC recurrieron a una táctica estratégica: inducir el enfrentamiento del vecino contra el vecino, lo que desgarró el tejido social. Como indica la Comisión de la Verdad de las Naciones Unidas (CEH, 1999), la campaña "tierra quemada" del gobierno guatemalteco resultó en más de 4,400 muertes en el municipio de Rabinal, aproximadamente el 20\% de su población.

En el emblemático caso de Río Negro, una serie de cuatro masacres cobró la vida de más de 
440 hombres, mujeres y niños, más de la mitad de la población (Colajocomo, 1999); un ejemplo clásico de la relación entre violencia y "desarrollo" (Alonso-Fradejas, 2012). Los habitantes de Río Negro fueron atacados por su oposición pacífica a su reubicación forzada para dar espacio a la hidroeléctrica de Chixoy, financiada por el Banco Mundial (Colajacomo, 1999). Los que sobrevivieron terminaron en el "pueblo modelo" de Pacux, en las afueras de Rabinal (Johnston, 2005). Sin tierra para cultivar y sin ninguno de los servicios inicialmente prometidos por el gobierno - por eso se resistían a irse- los sobrevivientes vivieron y viven en una situación precaria en condiciones de dependencia (Acevedo, 2004; Einbinder, 2017).

Aunque extremo, este caso muestra la actitud del Estado que percibe a los pueblos indígenas no conformes o "improductivos" como desechables, o como amenaza para el progreso de la nación (Kurtenbach, 2008). También ilustra el tipo de divisiones, empobrecimiento y desesperación provocadas por la violencia en varias comunidades achí (CIIDH, 1999).

No obstante, que la firma de los Acuerdos de Paz de 1996 produjo grandes esperanzas, la recuperación se encuentra estancada por la corrupción del gobierno y los enfoques neoliberales de desarrollo que priorizan el modelo de extracción/ exportación, ofreciendo poco a la mejora estructural de las zonas rurales (Robinson 2000; GranovskyLarsen, 2018). Las disparidades extremas, en particular en los ingresos y la propiedad de la tierra, además de los altos niveles de delincuencia, siguen sin ver el fin (Benson et al., 2008; Smith \& Offit, 2010). A pesar de los prósperos indicadores macroeconómicos (Cabrera et al., 2015), la pobreza, no solo en términos de riqueza acumulada, sino también de analfabetismo, seguridad alimentaria y acceso a los servicios, alcanza el $80 \%$ (CIDH, 2003). En un estudio reciente (Orozco \& Muñoz, 2016), los investigadores de Naciones Unidas constataron que casi la mitad de la población achí corre el riesgo de no disponer de recursos suficientes para comprar o cultivar alimentos adecuados, y una cuarta parte ya ha alcanzado ese límite.

En el territorio achí la situación es igualmente difícil, si no es que más, dada la fragmentación como resultado del conflicto y la alta vulnerabilidad con respecto al cambio climático (Acevedo, 2004; Stewart, 2006; SEGEPLAN, 2010). Como señalaron los dirigentes, el conflicto rompió todos los aspectos de la producción local y las relaciones sociales, que aún no han podido ser reconstruidas. Una combinación de condiciones económicas y agrícolas cada vez más difíciles, más un proceso de modernización que devalúa la producción en pequeña escala (Isakson, 2009), envía a masas de pequeños agricultores a las plantaciones para trabajar en los márgenes de la Ciudad de Guatemala o en Estados Unidos. En comunidades como Pacux, las condiciones llegan a su límite absoluto en términos de inseguridad alimentaria y desesperación, impulsadas por la falta de oportunidades económicas y el persistente trauma psicológico (Johnston, 2005; Einbinder, 2017).

\subsection{Agroecología en el territorio maya achí}

"Hemos perdido la esencia de nuestra identidad" Promotor agroecológico

De manera similar a lo documentado en otras partes del país (Fundebase, 2012; Reynolds, 2013), las organizaciones locales y externas han iniciado 
programas de desarrollo dentro del territorio achí, centrándose en la agroecología como medio para enfrentar las crisis de seguridad alimentaria y cultural mencionadas arriba, así como para fomentar la recuperación de la comunidad. El reconocimiento de la inmensa pérdida cultural ha dado lugar a la formación de asociaciones locales con el fin de recuperar prácticas ancestrales sostenibles, tales como la milpa diversificada, además de empoderar a las víctimas -en particular a las viudas-y motivar a la próxima generación de administradores de tierras.

Uno de los grupos más significativos es la asociación campesina Qachuu Aloom (QA), o "Madre Tierra", en achí. Fundada en 2002 por supervivientes de la masacre con la ayuda de un ciudadano estadounidense que vivía entonces en Rabinal, su intención fue rescatar los cultivos de alimentos y medicinas nativas mediante estableciendo huertos caseros diversificados y orgánicos, y bancos de semillas, a la vez que ofrecía a sus miembros incentivos económicos con la venta de sus productos. Desde entonces se ha ampliado a 25 comunidades achí, ha crecido a más de 600 miembros y despertado un alto nivel de interés. Con un enfoque continuo en la recolección y venta de semillas criollas y en el tratamiento de la seguridad alimentaria a nivel familiar, QA utiliza un "enfoque integrado", brindando asistencia técnica y abordando otras cuestiones como la desigualdad de género, la nutrición y la recuperación de determinadas prácticas y plantas tradicionales por medio de talleres y actividades de concienciación.

Pero QA y otras asociaciones locales no son las únicas que promueven la agroecología en la región. Desde mediados de los años noventa han llegado al territorio la FAO, Caritas, la alemana GIZ,
World Neighbor's, el Ministerio de Agricultura de Guatemala (MAGA) y muchas organizaciones más, con la intención de enseñar y alentar a los pequeños agricultores en las prácticas agroecológicas, como la conservación de suelos, la fabricación de abono orgánico, la producción de hortalizas, las estrategias de diversificación y la cría de animales. En años recientes, la investigación ha confirmado la expansión del "Corredor Seco", que actualmente absorbe los distritos de las tierras bajas del territorio, envía grupos e instituciones a la región, y se enfoca en la adaptación a la sequía y la gestión del agua (FAO, 2011).

Además de observar el éxito de los grupos locales y externos en cuanto a motivar a los agricultores para que adopten nuevas prácticas y cultivos y recuperen las ancestrales, reconocemos sus grandes diferencias en lo relacionado a ideologías y metodologías subsecuentes, lo que afecta la colaboración entre ambos, genera divisiones, e inhibe la eficacia de los proyectos individuales. En la siguiente sección detallamos cómo se manifiestan estas distinciones sobre la tierra.

\section{Resultados y discusión}

El objetivo de esta sección es ilustrar los procesos de escalamiento agroecológico en el territorio achí. Lo hacemos analizando temas emergentes de nuestro análisis de campo, esto es: la influencia de las organizaciones locales y externas, el cambio y la recuperación cultural/ambiental, la búsqueda de alternativas, y los beneficios económicos. 


\subsection{Organizaciones}

En primer lugar, nos ocupamos de los impactos de las organizaciones en los procesos de escalamiento agroecológico, con especial atención en los grupos externos y cómo se perciben en el territorio. Como parte del proyecto neoliberal de gran escala, las ONG e instituciones extranjeras llegaron a Guatemala después del conflicto para llenar los vacíos que había dejado un Estado progresivamente ausente, mientras intentaban inyectar su propia visión de alivio de la pobreza, la igualdad social y la protección del medio ambiente (Sundberg, 1998). En Rabinal, los grupos centrados en la agricultura sostenible llegan con el fin de trabajar con las personas y las comunidades con base en su disposición a colaborar, en su relación con el conflicto -por lo general, los más afectados reciben una atención adicional- y en la disponibilidad de recursos. Nuestras investigaciones pudieron constatar que la mayoría de los proyectos se relacionan con la seguridad alimentaria, la resistencia y la transición a métodos orgánicos, lo que se traduce en actividades agrícolas, como la fabricación de abonos orgánicos, el desarrollo de guarderías domésticas y bancos de semillas, y la experimentación con nuevas variedades de vegetales. Recientemente también se han identificado programas de recuperación de cultivos autóctonos altamente nutritivos, como el amaranto (Amaranthus), el chipilín (Crotalaria long irostrata) y la chaya (Cnidoscolus aconitifolius).

Casi todos los agricultores que entrevistamos reconocieron haber participado en algún momento con organizaciones externas. Las personas suelen recibir el apoyo de técnicos, que incluyen agrónomos de formación universitaria y promotores indígenas locales, y asisten a talleres, además de que reciben algún tipo de ayuda, como alimentos básicos, herramientas y pagos en efectivo. Algunos agricultores avanzados son promotores comunitarios delegados $\mathrm{y}$, aunque normalmente se trata de una tarea voluntaria, encontramos al menos un caso en el que hubo compensación económica.

Sería difícil decir la cantidad de campesinos que están cultivando agroecológicamente como resultado directo de su participación en organizaciones externas, en parte porque hay una labor similar desde las asociaciones locales con muchas de las mismas personas; sin embargo, creemos que es sustancial. Basamos este supuesto en las narraciones que escuchamos y en observaciones clave. En primer lugar, la producción de vegetales y hierbas no autóctonos -cebollas, hojas de mostaza, acelgas, remolacha, rábano, col, perejil y albahaca, entre otros- no existía en el territorio antes de la llegada de los grupos externos en el decenio de 1970, o se limitaba a muy pocas comunidades. Lo mismo ocurre con la elaboración de abono orgánico y otras prácticas de conservación del suelo. Estas técnicas, el mejoramiento natural del suelo y la producción de hortalizas para la subsistencia y las estrategias orientadas al mercado están muy difundidas y bien integradas en la producción familiar y la economía local. Además, hay varias iniciativas de cultivo de café orgánico y diversificado, en pequeña escala, que no existirían sin la asistencia de instituciones externas.

Pero a pesar de estos impactos en apariencia positivos, las narrativas sobre la presencia de grupos externos son en general pesimistas, sobre todo por parte de los líderes locales que abogan por la soberanía alimentaria. Coinciden con 
otras críticas a los programas gubernamentales y no gubernamentales para la agricultura de subsistencia e infrasubsistencia (Caballeros, 2013). Los defensores locales afirman que los proyectos iniciados por instituciones externas refuerzan las relaciones paternalistas y de dependencia. Y algunos van más allá, al afirmar que los grupos externos y sus proyectos "nos mantiene dentro del conflicto" pues conservan los antiguos desequilibrios de poder y representan nuevas formas de neocolonialismo. Un problema clave apunta a que la metodología incluye la entrega de alimentos $\mathrm{y} / \mathrm{u}$ otros regalos para los participantes que lo deseen. Esto lo consideran muy desmotivador; los agricultores dudan en transitar voluntariamente hacia la agroecología y la autosuficiencia "o hacer algo", como dijo un habitante, a menos que se les compense como es debido. Al igual que los productores se acostumbran a la facilidad de los agroquímicos, se vuelven dependientes del siguiente "proyecto", en el que la pobreza y el abandono del Estado pueden ser aprovechados para obtener el máximo beneficio económico.

Otras críticas incluyen la corta duración, que normalmente va de uno a dos años, y la impersonalidad de los programas externos, los métodos de enseñanza de arriba abajo o "extensionistas", así como las expectativas poco razonables. Según un líder, “... las organizaciones llegan y piensan que porque te compran una manguera, todo cambiará de la noche a la mañana... Vienen y van, gastan dinero en su proyecto $\mathrm{y}$ se van. Te conviertes en nada más que un número. No es así como funciona la naturaleza..." Además, señalaba otro líder, varios de estos grupos trabajan "con un pie en lo orgánico y el otro en lo convencional". Es decir, que mientras en algunos proyectos trabajan con mujeres para la agricultura orgánica, simultáneamente se distribuyen semillas de maíz "mejoradas" y paquetes tecnológicos a las contrapartes masculinas. Esto es polémico, dados los supuestos sobre la adaptabilidad de las semillas híbridas, los fuertes lazos culturales con las variedades locales, los temores sobre la contaminación genética, y el laborioso trabajo para educar sobre los riesgos asociados a los herbicidas, tanto en términos de efectos sobre la salud como de su amenaza para los cultivos nativos (LunaGonzalez \& Sørensen, 2018).

Si bien las asociaciones de base achí tienen objetivos similares -alentar a los agricultores a adoptar prácticas sostenibles para aumentar su autonomía y mejorar sus medios de vida, salvaguardar los recursos naturales y las prácticas/ variedades culturales-, sus metodologías son distintas. Fusionan la transferencia de conocimientos con el acompañamiento de largo plazo y la horizontalidad, como los intercambios entre agricultores. Y aunque son realistas en cuanto a la necesidad de incentivos económicos mediante la adopción de la agroecología, su labor suele presentarse fusionada con un componente espiritual/ cultural: la conexión con la naturaleza y Dios, y la revaloración de las prácticas ancestrales.

A pesar del éxito observados en la motivación para que en el territorio achí se adopten prácticas agroecológicas y se participe en programas integrados, la labor de estas comunidades está comprometida con los grupos externos, y perciben que "no pueden competir" con las metodologías "asistencialistas" o basadas en la ayuda. Asimismo, se quejan de la pereza y dependencia de los miembros acostumbrados a las limosnas: actitudes que no se corresponden del todo con el duro trabajo 
asociado a la producción agroecológica, por no hablar de los intercambios de conocimientos entre agricultores no remunerados. Por otra parte, los dirigentes locales refieren una constante batalla tanto con los políticos como con las empresas -que a menudo trabajan con el mismo objetivo-, que enganchan a los campesinos con los productos agroquímicos: fertilizantes sintéticos y herbicidas.

En conclusión, identificamos un entorno complejo en el que grupos locales y externos intentan llevar a cabo una labor similar pero con distintos principios. Y si pareciera que los métodos de los grupos no locales frustran los esfuerzos de las asociaciones achí, es posible que las barreras también vivan el efecto de las consecuencias persistentes del conflicto, entre las que se advierten las divisiones de la comunidad, los problemas domésticos, la pérdida de conocimientos y la dependencia respecto de los programas de restitución impulsados políticamente. Por último, puede ser importante comentar el papel adicional de los grupos internacionales en la financiación de las iniciativas locales de base, que, según nuestras observaciones, también causan complicaciones en cuanto a la dirección de determinados programas y la competencia entre los grupos participantes.

\subsection{Cambio y recuperación cultural/ambiental}

Un hecho notable es haber encontrado que en el territorio achí los procesos de recuperación comunitaria/cultural suelen estar entrelazados con iniciativas agroecológicas. Tanto las organizaciones locales como las externas capitalizan la aspiración de ciertos sectores de la población por recuperar prácticas ancestrales a fin de impulsar su agenda agroecológica, a la vez que intentan reparar el tejido social desgarrado. El programa de semillas iniciado por QA es un ejemplo de ello. Junto con otras sociedades agrícolas tradicionales, cabe destacar la importancia de las semillas entre los achíes, que resuena con cuestiones de identidad y autonomía local (Isakson, 2009). Este programa es estratégico porque ofrece a los participantes -principalmente mujeres-asistencia local experta en la recuperación de conocimientos y variedades, y proporciona un incentivo económico mediante la compra de semillas excedentes, que luego se revenden a la comunidad. El resultado es probablemente el programa agroecológico más exitoso del territorio en cuanto a número de participantes (más de 250 familias), así como en sus efectos generales directos e indirectos que incluyen la salvaguardia del material genético, la generación de ingresos familiares, el acercamiento de los vecinos en torno a objetivos comunes y la muy importante participación de los residentes en un nivel cada vez más profundo de siembra, cosecha y consumo de productos saludables.

Identificamos, por otra parte, el cambio cultural que, agravado por las alteraciones en las condiciones ambientales, es una barrera clave para la difusión y adopción de la agroecología. Más concretamente nos referimos a los valores cambiantes, en particular entre los jóvenes, y a cuestiones como la migración, el cambio climático y las sequías recurrentes, y a lo que muchos denominan la creciente "impracticabilidad de ser campesino". Este último punto deriva tanto de las condiciones económicas crecientemente difíciles como de las de crecimiento, y de la narrativa consistente y degradante que señala el trabajo de los 
pequeños agricultores como atrasado y antiprogreso (Loker, 1996).

En cuanto a la juventud local, los dirigentes expresan una gran urgencia por atraer y formar a la próxima generación de productores agroecológicos, ya que muchos profesionales están llegando a una edad en la que pronto se jubilarán. Y aunque las organizaciones están elaborando actualmente metodologías, incluidos programas escolares y actividades de fin de semana, la mayoría admite que convencer a los jóvenes de que "se enamoren del trabajo" es sumamente difícil. Esto se debe, en parte, al cambio observable en las prioridades, ahora centradas en ganar dinero y consumir, y a la "falsa esperanza de la educación [pública]", que, además de ser notablemente deficiente, adopta la estrategia neoliberal preparando a los estudiantes para que se conviertan en "profesionales", por ejemplo, en asalariados serviles para trabajos que no existen fuera de Ciudad de Guatemala (Poppema, 2009).

El legado del conflicto trae consigo todo un nuevo muestrario de barreras culturales. Los individuos comentan cómo el conflicto dio lugar a un "círculo de cambios", en el que la gente "deja de plantar, [y por lo tanto] pierde la comida regional, comprando lo que es barato y procesado..." Cabe señalar que la pérdida de conocimientos debido a la ruptura de los hábitos y costumbres, sumada a la creciente dependencia de los programas de restitución y ayuda, es evidente en casi todas las comunidades achí, algunas peor que otras.

Si bien reconocimos estas barreras, sabemos que muchos de los atributos negativos que engloban el contexto achí pueden convertirse en instigadores de la adopción. En la siguiente subsección demostramos cómo la identificación de los problemas y la búsqueda colectiva de soluciones pueden actuar como un determinante crítico para la participación en programas agroecológicos, al tiempo que incorpora los principios en los aspectos de su vida cotidiana.

\subsection{Búsqueda de alternativas}

Como señalan Mier y Terán et al. (2018), la identificación colectiva de una crisis es fundamental para generar un interés generalizado en la adopción agroecológica. En el territorio achí, la conciencia y la preocupación por diversas cuestiones ambientales y sociales, debido en parte a la labor educativa y de motivación de las organizaciones, se ha despertado el interés por los principios agroecológicos. En nuestras entrevistas los participantes expresan su temor por la creciente contaminación del agua, la deforestación, o el uso de plásticos y agroquímicos. Hablan de que enfermedades como el cáncer resultan de los cambios en la dieta y de la forma en que se cultivan sus verduras, por lo que entonces podrían elegir cultivar las suyas y no comprarlas en el mercado. Se acepta ampliamente que la obesidad es producto de los alimentos procesados, cuyo consumo a nivel nacional ha aumentado drásticamente en los últimos años (FAO, 2017). Una consecuencia es que se ha reportado un incremento en la reintroducción de plantas con alto contenido nutricional, tales como el amaranto y el chipilín, entre otras; además de los programas de educación popular para la nutrición. También se observan retroalimentaciones positivas, ya que la disminución del uso de herbicidas estimula el crecimiento natural de muchas de estas plantas en sus milpas, listas para ser consumidas y/o vendidas. Y con el aumento del interés en las plantas verdes, los productores 
agroecológicos encuentran más compradores, que, como se explica en la siguiente sección, buscan a los vecinos en lugar de comprar en el mercado.

Finalmente tocamos el tema del cambio climático, que para algunos productores que viven en las regiones más secas del territorio, ha significado el fin de la producción de milpa tal como la conocen. Como secuela, la creencia de una "sequía permanente" ha alejado a los potenciales colaboradores, al tiempo que ha atraído a otros convencidos de la capacidad innovadora de la agricultura agroecológica. En la granja de uno de los participantes observamos una transición de varios años de lo que una vez fue la milpa -lo que él describía como fuera de lugar, en las condiciones cambiantes- a lo que ahora es un “jardín permanente", con árboles frutales, pacaya (una palma comestible), café en el sotobosque y una gran cantidad de plantas comestibles semisalvajes que crecen debajo. Esto ejemplifica cómo la agroecología puede ser adoptada y promovida como parte de un mayor reconocimiento de que la "crisis" es inminente, y que aquellos que buscan una mayor autonomía, como la que tuvieron sus padres y abuelos, estarán mejor preparados, e incluso más contentos.

\subsection{Beneficios económicos}

En cuanto a los beneficios económicos, no podemos exagerar los impactos. "Plantamos para sobrevivir" es una frase que se escucha una y otra vez, en particular entre los que están privados de derechos y en situación de inseguridad alimentaria, con pocas opciones para llegar a fin de mes. Las prácticas agroecológicas pueden requerir más trabajo -desyerbar con herramientas manuales, construir la tierra mediante abono, cuidar de plantas perennes-, pero los participantes reconocen la posibilidad de eliminar los insumos costosos sin dejar de obtener una cosecha abundante. Los agricultores de escasos recursos también adoptan rápidamente estrategias de diversificación, ya que esto permite un ciclo continuo de cosecha.

La venta de pequeñas cantidades de cultivos o semillas excedentes aporta relativamente pocos ingresos, pero "es algo" en medio de una escasez de oportunidades económicas. Algunos productores agroecológicos ven la posibilidad de enviar a un niño a la escuela con el dinero extra ganado y ahorrado, y en el mejor de los casos, evitar el trabajo estacional en las granjas industriales de otras regiones. Los entrevistados hablan de la agricultura "con la naturaleza" en términos muy prácticos. Aprenden a elaborar abono con desechos de jardín y animales, o practican la rotación de cultivos "por necesidad", en contraste con motivos culturales y/o ambientales más matizados. Al incorporar las prácticas agroecológicas en sus actividades domésticas, los individuos "pueden no tener dinero, pero siempre algo que comer”, y mejor aún, "alimentos que no dañan el cuerpo".

Un número selecto de nuestros informantes convierten la producción agroecológica en empresas relativamente rentables, vendiendo en los mercados regionales y a los vecinos que "compran porque saben que no usamos veneno". Son innumerables los factores que contribuyen al éxito de estos emprendedores, pero pudimos observar la importancia de los recursos disponibles y la fuerte voluntad personal, a menudo relacionada con el deseo de continuar la labor de sus padres y abuelos, ya por motivos culturales o económicos o porque estos últimos se consideran sistemáticamente 
"más independientes". En dos comunidades en las que se ha registrado un interés excepcional en las actividades QA y las prácticas agroecológicas, las fuentes indican un liderazgo local de apoyo inusual que ha dado lugar a talleres de desarrollo comunitario y a programas de reforestación y protección de cuencas hidrográficas. Aunque prometedor, se dice que atrae el interés de grupos externos y del gobierno municipal, lo que provoca competencia, corrupción y dependencia entre los habitantes. En una comunidad específica -que a menudo se le reconoce como la "joya de la corona" de la producción orgánica regional por sus diversos jardines y la protección del agua/bosque-, las opiniones sobre los habitantes "altamente individualistas" y sus motivos "capitalistas", así como de dependencia del apoyo externo y la falta de integración de principios más sociales, son expresadas por los líderes y señaladas de primera mano. Una vez más, esto toca el papel crítico de las organizaciones al lado de la espinosa cuestión de lo que puede y no puede determinarse como "agroecología" (Wezel \& Soldat, 2009; Giraldo \& Rosset, 2017).

En resumen, hallamos una situación extremadamente compleja con numerosas contradicciones y factores por explorar. Algunas características parecen compatibles con lo que ha recogido la literatura, como la identificación de una crisis y las prácticas efectivas. Otras, en específico las que tienen que ver con la falta de un tejido social fuerte y de políticas públicas, van en contra de las expectativas y merecen un mayor examen, mismo que hemos intentado aquí. Es de especial importancia la influencia de las organizaciones, tanto locales como externas, cuyos impactos son tanto positivos como negativos.

\section{Conclusión}

Nuestro objetivo en este texto ha sido destacar los procesos y el potencial del escalamiento agroecológico en condiciones difíciles y subóptimas; y más concretamente, abrir nuevos puntos de discusión y debate respecto a los factores clave o "impulsores" identificados en la literatura, muchos de los cuales están ausentes o mínimamente representados en el caso achí.

Para concluir, planteamos una pregunta: ¿puede ocurrir un escalamiento agroecológico en la "periferia", como se ejemplifica en el territorio achí? Según nuestro análisis, es posible. Esto se debe en gran medida a una búsqueda común de mayor independencia o autonomía (Van der Ploeg, 2008) por parte de la comunidad achí, junto con la determinación de algunos de sus sectores para recuperar los atributos culturales dañados o perdidos durante el conflicto, en particular los relativos a las prácticas agrícolas y las variedades locales. Si bien hacemos énfasis en la importancia de las organizaciones, a la par que la necesidad de debates más matizados sobre sus repercusiones, también reconocemos que la difusión de la agroecología en esta región puede estar igualmente determinada por la voluntad personal, las creencias espirituales, los antecedentes y la posición en relación con las oportunidades. Algo notable que atestiguamos en el caso achí es que se vincula con barreras aparentemente insuperables y con la forma en que se enfrentan con determinación, interés y optimismo continuos. Como acotaba un dirigente, no es tan determinante si los individuos están plantando grandes extensiones agroecológicas, sino que cada vez más personas se involucran, a cualquier nivel 
que puedan permitirse en ese momento. Si esto es lo que mide el éxito, entonces nos sentimos optimistas por la actividad presente y las posibilidades futuras.

En lo relativo a ulteriores investigaciones sobre el tema, hemos identificado una gama de posibilidades en lo que respecta a estudios más profundos sobre los impactos de las organizaciones externas y la dependencia, y si ciertas formas de asistencia pueden impulsar los movimientos iniciales de escalamiento. Del mismo modo, esperamos que se realicen más trabajos sobre los enfoques metodológicos, en especial porque tienen en cuenta las condiciones y limitaciones sociales/ políticas específicas. De interés adicional sería el desarrollo de vínculos claros entre la cultura/cambio cultural y los procesos de escalamiento, que también se presentan incompletos en la literatura relevante.

\section{Agradecimientos}

Estamos profundamente agradecidos y en deuda con los promotores y las promotoras de Qachuu Aloom y con los participantes, así como con los numerosos líderes comunitarios que nos ayudaron a llevar a cabo este trabajo. Sin su paciencia y asistencia nada de esto hubiera sido posible. Asimismo agradecemos a Marc Edelman y Peter Rosset por sus valiosos comentarios y aportaciones.

\section{Referencias}

Acevedo, S. Las viudas del conflicto armado en Rabinal. Estrategias de sobrevivencia en el contexto de la pobreza. Guatemala City, Tesis (Maestría del Programa Multidisciplinario de Estudios sobre la Pobreza) - FLACSO Guatemala, 2004.

Alonso-Fradejas, A. Land control-grabbing in Guatemala: the political economy of contemporary agrarian change. Canadian Journal of Development Studies, 33(4), 509-528, 2012. doi: 10.1080/02255189.2012.743455

Altieri, M. A. Agroecology: the scientific basis of alternative agriculture. Boulder, CO: Westview Press, 1987.

Altieri, M. A.; Toledo, V. M. The agroecological revolution in Latin America: Rescuing nature, ensuing food sovereignty and empowering peasants. The Journal of Peasant Studies, 38(3), 2011. doi: 10.1080/03066150.2011.582947

Ball, P.; Kobrack, P.; Sprirer, H. F. State violence in Guatemala, 1960-1996: a quantitative reflection. Washington, DC: American Association for the Advancement of Science (AAAS), 1999.
Baxter, J.; Eyles, J. Evaluating qualitative research in social geography: establishing 'rigour' in interview analysis. Transactions of the Institute of British Geographers, 22, 505-525, 1997. doi: 10.1111/tran.1997.22.issue-4

Benson, P.; Fischer, E. F.; Thomas, K. Resocializing suffering: neoliberalism, accusation, and the sociopolitical context of Guatemala's new violence. Latin American Perspectives, 35(5), 35-58, 2008. doi: 10.1177/0094582X08321955

Brescia, S. (Ed.). Fertile ground: scaling agroecology from the ground up. Oakland, CA: Food First Books, 2017.

Caballeros, Á. Agricultura familiar, soberanía alimentaria y buen vivir: alternativos y desafíos en Guatemala. Guatemala: Magna Terra Editores, Serie Cuadernos Populares, n. 2, 2013.

Cabrera, M.; Nora, L.; Hilcías, M. Fiscal policy, inequality, and the ethnic divide in Guatemala. World Development, 76, 263-279, 2015.

Carmack, R. M. (Ed.). Harvest of violence: the Maya Indians and the Guatemala crisis. Norman: University of Oklahoma Press, 1988. 
CEH - Comisión para el Esclarecimiento Histórico. Guatemala: memoria del silencio. Report of the Commission for Historical Clarification Conclusions and Recommendations. Guatemala: CEH, 1999.

CIDH - Comisión Interamericana de Derechos Humanos. La situación de los Pueblos Indígenas. En: CIDH. Justice inclusión social: los desafíos de la democracia en Guatemala. Washington D.C.: OEA, 2003. Disponible en: http://www. cidh.oas.org/pdf\%20files/GUATEMALA.2003.pdf

CIIDH - International Center for Human Rights Research. Draining the sea: an analysis of terror in three rural communities in Guatemala (1980-1984). Guatemala: American Association of the Advancement of Sciences, 1999.

Colajacomo, J. The Chixoy Dam: the Maya Achi' genocide. The story of forced resettlement. Contributing Paper. Cape Town, Sudáfrica: World Commission on Dams, 1999.

Denevan, W. M. 2 Prehistoric agricultural methods as models for sustainability. Advantage of Plant Pathology, 11, 21-43, 1995.

DeWalt, K. M.; DeWalt, B. R. Participant observation: a guide for fieldworkers. Lanham, Md: Rowman \& Littlefield, 2011.

Dunn, K. Interviewing. En: Hay, I. (Ed.). Qualitative research methods in human geography. Oxford, UK: Oxford University Press, p. 50-82, 2005.

Einbinder, N. Dams, development, and displacement: perceptions from Río Negro, Guatemala. Nueva York, NY: Springer/Palgrove, Springer Briefs in Latin American Studies, 2017.

FAO - Food and Agriculture Organization. El cambio climático en el corredor seco de Guatemala: experiencias en Baja Verapaz. Popayán, CO: FAO, 2011. Disponible en: https://www.fao.org/climatechange/26262-0eb7992915b69 48cf91ed2d0d7cbf7f49.pdf

FAO - Food and Agriculture Organization. El consumo de productos ultraprocesadas aumenta en Guatemala. Noticias, 2017. Disponible en: http://www.fao.org/ guatemala/noticias/detail-events/en/c/854050/
Fundebase. Politicas agrarias, agricolas y de desarrollo rural en Guatemala: del despojo histórico, la descampesinización actual y grandes luchas sociales desde el poder indigenacampesino por la tierra, el territorio y la vida. Guatemala: Alianza por la Agroecología, 2012.

Giraldo, O. F.; Rosset, P. M. Agroecology as a territory in dispute: between institutionality and social movements. Journal of Peasant Studies, 45, 1-20, 2017.

Gliessman, S. R. Agroecology: the ecology of sustainable food systems. Boca Raton, USA: CRC Press, 3. ed., 2015.

Gliessman, S. R.; Rosemeyer, M. The conversion to sustainable agriculture: principles, processes, and practices. Boca Raton, USA: Taylor \& Francis Group, 2010.

González, H. Agroecological reconfiguration: local alternatives to environmental degradation in Mexico. Journal of Agrarian Change, 12(4), 484-502, 2012. doi: 10.1111/ joac.2012.12.issue-4

Granovsky-Larsen, S. Land and reconfiguration of power in post-conflict Guatemala. En: North, L. L.; Clark, T. D. (Eds.). Dominant elites in Latin America. Latin America political economy series. Suiza: Palgrave Macmillan, p. 181-204, 2018.

Guzmán, C. G. I.; Alonso, M. A. M. La investigación participativa en agroecología: Una herramienta para el desarrollo sustentable. Ecosistemas, 16(1), 24-36, 2007.

Hainzelen, E. Enhancing the function and provisioning of ecosystem services in agriculture: Agroecological principles. En: Proceedings of the FAO international symposium, Agroecology for food security and nutrition. Rome, 18-19 sep., 2014.

Holt-Giménez, E. Measuring farmers' agroecological resistance after Hurricane Mitch in Nicaragua: a case study in participatory, sustainable land management impact monitoring. Agriculture Ecosystems \& Environment, (93), 87-105, 2002. doi: 10.1016/S0167- 8809(02)00006-3

Holt-Giménez, E. Campesino a campesino. Oakland, CA: Food First Books, 2006.

IDEAR - Institutos de Estudios Agrarios y Rurales. Nuestro maiz, nuestro futuro. Estudios para la reactivación de la 
producción nacional de maíz en Guatemala. Guatemala: Magna Terra, 2010.

Isakson, R. S. No hay ganancia en la milpa: the agrarian question, food sovereignty, and the on-farm conversation of agrobiodiversity in the Guatemalan Highlands. Journal of Peasant Studies, 36(4), 725-59, 2009. doi: 10.1080/03066150903353876

Johnston, B. R. Chixoy dam legacy issues study. executive summary: consequential damages and reparation: Recommendations for remedy. Santa Cruz, California: Center for Political Ecology, 2005.

Jonas, S. The battle for Guatemala: Rebels, death squads and U.S. power. Nueva York: Westview Press, 1991.

Khadse, A.; Rosset, P. M.; Morales, H.; Ferguson, B. G. Taking agroecology to scale: The zero budget natural farming peasant movement in Karnataka, India. The Journal of Peasant Studies, 24, 1-28, 2017.

Kremen, C.; A. Iles, A.; Bacon, C. Diversified farming systems: An agroecological, systems-based alternative to modern industrial agriculture. Ecology and Society, 17(4), 44, 2012. doi:10.5751/ES-05103-170444.

Kurtenbach, S. Guatemala's post-war development: the structural failure of low intensity peace. Institute for Development and Peace (INEF). Alemania: University of Duisburg-Essen, Project working paper no. 3, 2008.

Loker, W. M. "Campesinos" and the crisis of modernization in Latin America. Journal of Political Ecology, 3, 69-88, 1996. doi: $10.2458 / \mathrm{v} 3 \mathrm{i} 1.21774$

Lovell, W. G. Surviving conquest: the Maya of Guatemala in historical perspective. Latin American Research Review, 23(2), 25-57, 1988.

Luna-González, D.; Sørensen, M. Higher agrobiodiversity is associated with improved dietary diversity, but not child anthropometric status, of Mayan Achí people of Guatemala. Public Health Nutrition, 11, 2128-2141, 2018.

Machín Sosa, B.; Jaime, A. M. R.; Lozano, D. R. Á.; Rosset, P. M. Agroecological revolution: the farmer-tofarmer movement of the ANAP in Cuba. La Habana: ANAP and La Vía Campesina, 2013. Disponible en: https://
viacampesina.org/downloads/pdf/en/Agroecologicalrevolution-ENGLISH.pdf

McCune, N.; Sánchez. M. Teaching the territory: agroecological pedagogy and popular movements. Agriculture and Human Values, 1-16, 2018. doi: 10.1007/ s10460-018-9853-9

Mier y Terán Giménez Cacho, M.; Giraldo, O. F.; Aldasoro, M.; Morales, H.; Ferguson, B. G.; Rosset, P.; Khadse, A.; Campos, C. Bringing agroecology to scale: key drivers and emblematic cases. Agroecology and Sustainable Food Systems, 42(6), 637-665, 2018. doi: 10.1080/21683565.2018.1443313

Morales, H.; Perfecto, I. Traditional knowledge and pest management in the Guatemalan highlands. Agriculture and Human Values, 17, 49-63, 2000. doi: 10.1023/A:1007680726231

Nicholls, C. I.; Altieri, M. A.; Vasquez, L. Agroecology: principles for the conversión and redesign of farming systems. Journal of Ecosystem and Ecography, 5(S5), 10, 2016.

Orozco, A.; Muñoz, G. Bajos ingresos agobian al 82 por ciento de la población. Prensa Libre, 6 oct., 2016. Parmentier, S. Scaling-up agroecological approaches: what, why and how? Bélgica: Oxfam Solidarity, 2014.

Perfecto, I.; Vandermeer, J. Coffee agroecology. New York, NY: Routledge, 2015.

Perfecto, I.; Vandermeer, J.; Wright, A. L. Nature's matrix: linking agriculture, conservation and food sovereignty. London: Earthscan, 2009.

Poppema, M. Guatemala, the peace accords and education: a post-conflict struggle for equal opportunities, cultural recognition and participation in education. Globalisation, Societies and Education, 7(4), 383-408, 2009. doi: 10.1080/14767720903412218

Pretty, J. Agri-culture: reconnecting people, land and nature. London: Earthscan, 2002.

Reynolds, L. Guatelama: the long battle to preserve ancestral farming practices. Eurasia Review, 2013. 
Robinson, W. I. Neoliberalism, the global elite, and the Guatemalan transition: a critical macrosocial analysis. Journal of Interamerican Studies and World Affairs, 42(4), 89-107, 2000. doi:10.2307/166343.

Rosset, P. Social organization and process in bringing agroecology to scale. Agroecology for food security and nutrition. En: Proceedings of the FAO International Symposium. Rome, 18-19 sept., 298-307, 2014.

Rosset, P. M.; Martínez-Torres, M. E. Rural social movements and agroecology: context, theory, and process. Ecology and Society, 17(3), 17, 2012. doi: 10.5751/ES05000-170317

Sarandón, S. J.; Flores, C. C. (Eds.). Agroecología: bases teóricas para el diseño y manejo de agroecosistemas sustentables. La Plata, Argentina: Universidad de La Plata, 2014.

SEGEPLAN - Secretaría de Planificación y Programación de la Presidencia, Guatemala. Plan de Desarrollo Municipal-PDM de Rabinal, Baja Verapaz, Guatemala 2011- 2025, 132. Guatemala: Government Report, 2010.

Smith, T. J.; Offit, T. A. Confronting violence in postwar Guatemala: an introduction. The Journal of Latin American and Caribbean Anthropology, 15(1), 1-15, 2010. doi: 10.1111/j.1935-4940.2010.01060.x

Steinberg, M. Maize diversity and cultural change in a Maya agroecological landscape. Journal of Ethnobiology, 19(1), 127-139, 1999.

Stewart, J. When local troubles become transnational: The transformation of Guatemalan indigenous rights movement. En: Johnston, H.; Alemida, P. (Eds.). Latin American social movements: globalization, democratization, and transnational networks. Maryland, USA: Rowman and Littlefield, p. 259-278, 2006.

Sundberg, J. NGO landscapes: conservationand communities in the Maya Biosphere Reserve, Peten, Guatemala. Geographical Review, (88), 388-412, 1998. doi: $10.2307 / 216016$.

Tedlock, D. Rabinal achi: a Mayan drama of war and sacrifice. Oxford, UK: Oxford University Press, 2003.

Van der Ploeg, J. D. The new peasantries: struggles for autonomy and sustainability in an era of empire and globalization. London: Earthscan, 2008.

Weis, T. The global food economy: the battle for the future of farming. London: Zed books, 2007.

Wettasinha, C.; Waters-Bayer, A.; Veldhuizen, L. van; Quiroga, G.; Swaans, K. Study on impacts of farmerled research supported by civil society organizations. Penangy, Malaysia: CIGAR Research Programo on Aquatic Agricultural Systems. Working Paper: AAS, 2014.

Wezel, A.; Bellon, S.; Doré, T.; Francis, C.; Vallod, D.; David, C. Agroecology as a science, a movement and a practice. A Review. Agronomy for Sustainable Development, 29(4), 503-515, 2009. doi: 10.1051/agro/2009004

Wezel, A.; Soldat, V. A quantitative and qualitative historical analysis of the scientific discipline of agroecology. International Journal of Agricultural Sustainability, 7(1), 3-18, 2009. doi:10.3763/ijas.2009.0400

Wilken, G. C. Good farmers: Traditional agricultural resource management in Mexico and Guatemala. Berkeley: University of California Press, 1987. 